\title{
CIRCUIT PARTITIONS AND THE HOMFLY POLYNOMIAL OF CLOSED BRAIDS
}

\author{
FRANCOIS JAEGER
}

\begin{abstract}
We present an expansion of the homfly polynomial $P(D, z, a)$ of a braid diagram $D$ in terms of its circuit partitions. Another aspect of this result is an expression of $P(D, z, a)$ as the trace of a matrix associated to $D$ in a simple way. We show how certain degree properties of the homfly polynomial can be derived easily from this model. In particular we obtain that if $D$ is a positive braid diagram on $n$ strings with $w$ crossings, the maximum degree of $P(D, z, a)$ in the variable $a$ equals $n-1-w$.

Resume. Nous présentons une expansion pour le polynôme homfly $P(D, z, a)$ d'un diagramme de tresse $D$ en termes de ses partitions en circuits. Un autre aspect de ce résultat consiste en une expression de $P(D, z, a)$ comme trace d'une matrice associee de façon simple à $D$. Nous montrons comment certaines propriétés de degré du polynôme homfly dérivent simpiement de ce modèle. En particulier nous obtenons que pour un diagramme de tresse positif $D$ à $n$ brins et $w$ croisements, le degré maximum de $P(D, z, a)$ en la variable $a$ est égal à $n-1-w$.
\end{abstract}

\section{INTRODUCTION}

The homfly polynomial of an oriented link, whose discovery is presented in [7 and 22], can be defined inductively in a purely combinatorial way on link projections or diagrams. See $[9,18,22]$. This raises the question of the existence of a combinatorial model for this polynomial, similar to the models obtained by Kauffman for the special cases of the Alexander-Conway polynomial [14] and the Jones polynomial [15]. Such a model should lead to new, efficient proof techniques. This was already the case with Kauffman's "state model" for the Jones polynomial, as illustrated in [15, 21, and 23].

Important progress was made with the discovery of "state models" for an infinite sequence of one-variable specializations of the homfly polynomial [13, $16,17,24]$. A state model valid only for braid diagrams was also described in [10]. We present here another state model for the homfly polynomial of braid diagrams that appears to be simpler than the previous model of [10], and that yields straightforward explanations for some degree properties such as the

Received by the editors September 29, 1988 and, in revised form. February 23, 1989.

1980 Mathematics Subject Classification (1985 Revision). Primary 57M25; Secondary 57M15.

Key words and phrases. Links, braids, diagrams, graphs, polynomial invariants, combinatorial models, transfer matrices.

This research was supported in part by the PRC "Mathématiques et Informatique".

(C) 1991 American Mathernatical Society $0002-9947 / 91 \$ 1.00+\$ .25$ per page 
Morton-Franks-Williams inequalities [6, 19]. Quite recently, a model for the homfly polynomial of arbitrary diagrams that is similar to the one presented here was obtained in [11].

In $\S 2$ we introduce the basic concepts and terminology relative to link diagrams and the homfly polynomial. We present our main result in $\S 3.1$. This result is then reformulated in matrix terms in $\S 3.2$. Both formulations are used in the proof given in $\S 3.3$. Finally, some consequences of the model are derived in $\S 4$. For instance, for positive braids we show that equality holds in one of the Morton-Franks-Williams inequalities and we give a simple combinatorial interpretation of the coefficients of the Alexander-Conway polynomial.

\section{BASIC CONCEPTS}

2.1. Links, braids, and their diagrams. For the usual terminology of Graph Theory and Knot Theory, the reader is referred to [2-4 and 14]. By link we mean a tame, oriented link in the 3-dimensional Euclidean space. Links are represented by regular plane projections, which we call diagrams. We shall consider diagrams as 4-regular directed plane graphs whose vertices are signed. More precisely, a diagram is a triple $D=(V, E, s)$, where

- Some subset $E^{\prime}$ of $E$ is the edge-set of a plane directed graph $G$ in the usual sense (allowing loops and parallel edges) with vertex-set $V$.

- $E-E^{\prime}$ is a set of simple, oriented, closed Jordan curves in the plane that are disjoint from $G$ and mutually disjoint. We call these curves free loops. We consider $(V, E)$ as a graph with vertex-set $V$ and edge-set $E$, so that free loops are considered as edges without end-vertices.

- For each vertex $v$ there are exactly two edges with terminal end $v$ (they will be said incident into $v$ ) and exactly two edges with initial end $v$ (they will be said incident from $v$ ). Moreover, the two edges incident into $v$ form an angle at $v$.

- $s$ is a mapping from $V$ to $\{1,-1\}$ that associates to every vertex its sign. The sign mapping describes the crossing structure of the link represented by $D$ as shown in Figure 1 .

The writhe of a diagram $D$, denoted by $w(D)$, is the sum of the signs of its vertices.

Two diagrams will be said to be isotopic if they represent the same link up to ambient isotopy. The isotopy relation on diagrams is described combinatorially by Reidemeister's Theorem (see for instance, [4, Chapter 1]).
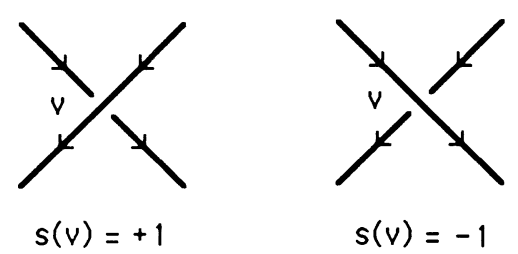

Figure 1 
Let us consider an infinite alphabet $\left\{s_{i}, i \in \mathbb{N}-\{0\}\right\}$. Artin's braid group on $n$ strings $B_{n}(n \geq 1)$ is defined by the presentation

$$
\left\langle s_{1}, \ldots, s_{n-1}\left|s_{i} s_{i+1} s_{i}=s_{i+1} s_{i} s_{i+1}, i=1, \ldots, n-2 ; s_{i} s_{j}=s_{j} s_{i},\right| i-j \mid \geq 2\right\rangle .
$$

Thus, $B_{n}$ is the subgroup of $B_{n+1}$ generated by $s_{1}, \ldots, s_{n-1}$ (which is trivial for $n=1$ ). We call braid (on $n$ strings) a pair $(b, n)$, where $b$ belongs to $B_{n}$. We introduce a special symbol $\varepsilon$ to represent the identity element of the groups $B_{n}$. We call a braid word on $n$ strings any nonempty word on the alphabet $\{\varepsilon\} \cup\left\{s_{i}, s_{i}^{-1} \mid i=1, \ldots, n-1\right\}$. Note that a braid word on $n$ strings is also a braid word on $n^{\prime}$ strings for all $n^{\prime} \geq n$. To every braid word $m=m_{1} \cdots m_{k}$ of length $k$ on $n$ strings we associate a braid diagram on $n$ strings as follows. For $i=0, \ldots, k$ and $j=1, \ldots, n$ let $p_{i, j}$ be the point in the $(x, y)$-plane with coordinates $(j,-i)$. To each letter $m_{i}$ $(i=1, \ldots, k)$ corresponds a portion of diagram situated in the rectangle with corners $p_{i-1,1}, p_{i-1, n}, p_{i, 1}, p_{i, n}$ according to the rule described in Figure 2.
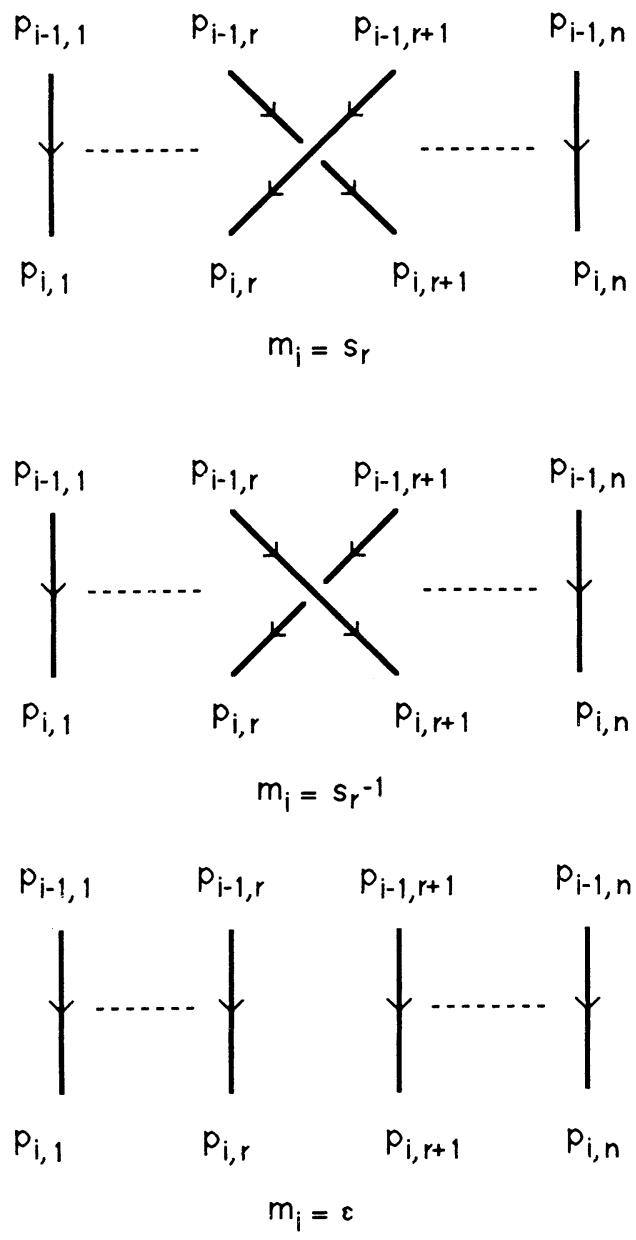

Figure 2 


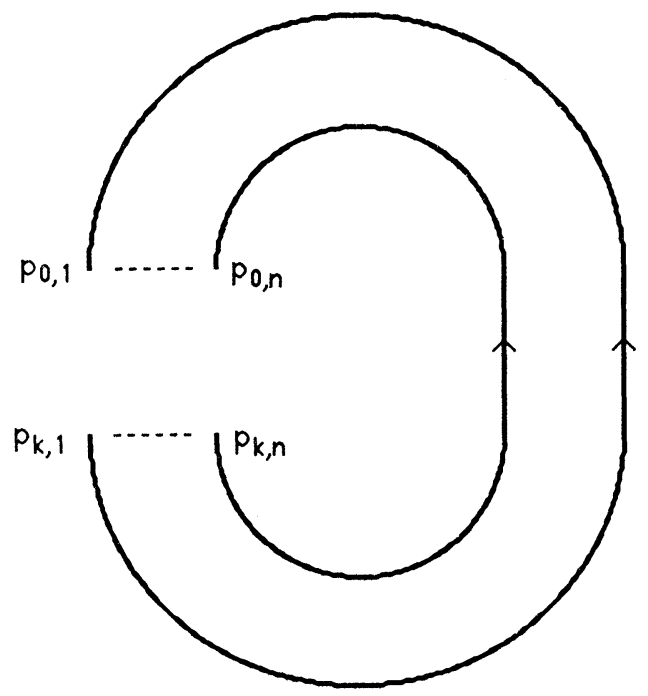

FIGURE 3

Now for each $j=1, \ldots, n$ we join the points $p_{k, j}$ and $p_{0, j}$ as shown in Figure 3 to form a diagram $D$. The edge of $D$ which contains the points $p_{k, j}$ and $p_{0, j}$ will be called a return edge and will be assigned the index $j$.

Let $(b, n)$ be a braid on $n$ strings. Let us choose a braid word on $n$ strings that represents $b$. The associated braid diagram on $n$ strings will be called a closure of $(b, n)$. It follows from the definition of the braid group $B_{n}$ that any two closures of $(b, n)$ are isotopic.

There exists a homomorphism $w$ from $B_{n}$ to the additive group of integers such that $w\left(s_{r}\right)=1$ and $w\left(s_{r}^{-1}\right)=-1 \quad(r=1, \ldots, n-1)$. Clearly if $D$ is a closure of $(b, n), w(b)$ equals $w(D)$, the writhe of $D$.

Two basic results on braid diagrams are the following.

Alexander's Theorem. Every diagram is isotopic to some braid diagram.

Markov's Theorem. Two braids have isotopic closures if and only one can be obtained from the other by a finite sequence of moves of one of the following types:

- Markov move of type 1: Replace $(b, n)$ by $\left(c b c^{-1}, n\right)$ (for $b, c$ in $\left.B_{n}\right)$

- Markov move of type 2: For $b$ in $B_{n}$, replace $(b, n)$ by $\left(b s_{n}, n+1\right)$ or $\left(b s_{n}^{-1}, n+1\right)$, or perform the converse operation.

2.2. The homfly polynomial. If $D^{+}, D^{-}$, and $D^{\circ}$ are diagrams that are identical outside a small disk and behave as depicted in Figure 4 inside the disk, we shall say that $\left(D^{+}, D^{-}, D^{\circ}\right)$ form a Conway triple.

The following result appears in [7, 9, 18, and 22]; see also [8, 12]. 


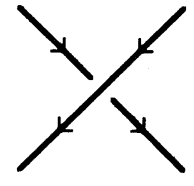

$D^{+}$

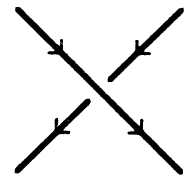

$D^{-}$

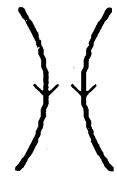

$D^{\circ}$

FIGURE 4

Proposition 1. One can associate (in a unique way) to every diagram $D$ a Laurent polynomial with integer coefficients in two variables, $z$ and $a$, which we denote by $P(D, z, a)$ in such a way that the following properties hold:

(i) If $D$ and $D^{\prime}$ are isotopic, $P(D, z, a)=P\left(D^{\prime}, z, a\right)$.

(ii) If $\left(D^{+}, D^{-}, D^{\circ}\right)$ form a Conway triple then

$$
a P\left(D^{+}, z, a\right)-a^{-1} P\left(D^{-}, z, a\right)=z P\left(D^{\circ}, z, a\right) .
$$

(iii) If $D$ consists of a single free loop, $P(D, z, a)=1$.

The polynomial $P$ is usually called the homfly polynomial or the JonesConway polynomial. We shall use the first terminology here.

Proposition 1 can be proved purely combinatorially by working in the class of all diagrams (see $[9,18,22])$. It can also be proved by working in the restricted class of braid diagrams, which allows the efficient use of algebraic methods (see $[8,12,25])$. We now present a simple consequence of this second approach.

In the sequel we denote by $K$ the ring of Laurent polynomials with integer coefficients in two variables, $z$ and $a$.

Proposition 2. For every positive integer $n$ let $F_{n}$ be a mapping from $B_{n}$ to $K$ and assume that the following properties hold:

(i) For all $b, b^{\prime}$ in $B_{n}, F_{n+1}\left(b s_{n} b^{\prime}\right)=F_{n+1}\left(b s_{n}^{-1} b^{\prime}\right)=F_{n}\left(b b^{\prime}\right)$.

(ii) For all $b, b^{\prime}$ in $B_{n}$ and $i=1, \ldots, n-1, a F_{n}\left(b s_{i} b^{\prime}\right)-a^{-1} F_{n}\left(b s_{i}^{-1} b^{\prime}\right)=$ $z F_{n}\left(b b^{\prime}\right)$.

(iii) $F_{1}(1)=1$.

Then for every positive integer $n$ and every closure $D$ of the braid $(b, n)$, $F_{n}(b)=P(D, z, a)$.

Proof. For every $n \geq 1$ and $b$ in $B_{n}$, let $D$ be a closure of $(b, n)$ and let $F_{n}^{\prime}(b)=P(D, z, a)$. Since any two closures of $(b, n)$ are isotopic, it follows from property (i) of Proposition 1 that $F_{n}^{\prime}(b)$ does not depend on the choice of $D$. Thus, we have defined for every $n \geq 1$ a mapping $F_{n}^{\prime}$ from $B_{n}$ to $K$. For all $b, b^{\prime}$ in $B_{n},\left(b s_{n} b^{\prime}, n+1\right)$ and $\left(b s_{n}^{-1} b^{\prime}, n+1\right)$ can each be obtained from $\left(b b^{\prime}, n\right)$ by one Markov move of type 2 inserted between two Markov moves of type 1. It then follows from Markov's Theorem and from property (i) of Proposition 1 that $F_{n+1}^{\prime}$ and $F_{n}^{\prime}$ together satisfy property (i) of Proposition 2. Similarly for all $i=1, \ldots, n-1$, we may choose closures of $\left(b s_{i} b^{\prime}, n\right)$, $\left(b s_{i}^{-1} b^{\prime}, n\right)$ and $\left(b b^{\prime}, n\right)$ that form a Conway triple. It follows from Property 
(ii) of Proposition 1 that $F_{n}^{\prime}$ satisfies property (ii) of Proposition 2. Finally, $F_{1}^{\prime}(1)=1$ is an immediate consequence of property (iii) of Proposition 1.

For every $n \geq 1$, let $H_{n}$ be the quotient of the group algebra $K\left(B_{n}\right)$ by the two-sided ideal $J_{n}$ generated by the elements $a s_{i}-a^{-1} s_{i}^{-1}-z \quad(i=1, \ldots, n-1)$ ( $H_{n}$ is isomorphic to the formal Hecke algebra $H_{n}$ of [25] and to the Hecke algebra $H(q, n)$ of [12], the difference consisting only of the choice of the parameters $a$ and $z$ ). The inclusion of $B_{n}$ in $B_{n+1}$ extends to an inclusion of $H_{n}$ in $H_{n+1}$. Let us extend $F_{n}$ and $F_{n}^{\prime}$ by linearity to $K\left(B_{n}\right)$. Then by property (ii) of Proposition 2 which is valid for $F_{n}$ and $F_{n}^{\prime}$, the kernels of $F_{n}$ and $F_{n}^{\prime}$ contain $J_{n}$. Thus $F_{n}$ and $F_{n}^{\prime}$ pass to the quotient and will be identified with the corresponding mappings from $H_{n}$ to $K$.

We now prove by induction on $n$ that $F_{n}=F_{n}^{\prime}$. This is true for $n=$ 1. Assume now that the result holds for the integer $n \geq 1$. We shall use the following classical result: $H_{n+1}$ is generated as a $K$-algebra by $G_{n+1}=$ $H_{n} \cup\left\{x s_{n} y / x, y \in H_{n}\right\}$ (see, for instance, [8, 12, 25]). Thus, it is enough to show that $F_{n+1}$ and $F_{n+1}^{\prime}$ coincide on $G_{n+1}$. For $x, y$ in $H_{n}$, it follows from property (i) of Proposition 2 and from the induction hypothesis that $F_{n+1}\left(x s_{n} y\right)=F_{n}(x y)=F_{n}^{\prime}(x y)=F_{n+1}^{\prime}\left(x s_{n} y\right)$. Finally properties (ii) and (i) yield $z F_{n+1}(x)=a F_{n+1}\left(x s_{n}\right)-a^{-1} F_{n+1}\left(x s_{n}^{-1}\right)=a F_{n}(x)-a^{-1} F_{n}(x)$ and the similar equality with $F^{\prime}$ instead of $F$. Then the equality $F_{n+1}(x)=F_{n+1}^{\prime}(x)$ follows by induction.

\section{A STATE MODEL FOR THE HOMFLY POLYNOMIAL OF BRAID DIAGRAMS}

3.1. Admissible circuit partitions. Let $\sigma$ be a permutation of a finite set $X$. The number of cycles of $\sigma$ will be denoted by $c(\sigma)$. Let $X^{\prime}$ be a subset of $X$ that contains at least one element of each cycle of $\sigma$. Such a subset $X^{\prime}$ together with a total ordering of its elements will be called an ordered transversal of $\sigma$. The standard word of $\sigma$ with respect to its ordered transversal $X^{\prime}$ is obtained as follows. For each cycle $C$ of $\sigma$ we denote by $g(C)$ the minimum element of $X^{\prime} \cap C$. Then we associate to $C$ the word $W(C)=x_{1} \cdots x_{|C|}$, where $x_{i}=\sigma^{i-1}(g(C))$ for $i=1, \ldots,|C|$. Now we denote the cycles of $\sigma$ by $C_{1}, \ldots, C_{c(\sigma)}$ in such a way that $g\left(C_{i}\right)$ is a smaller element of $X^{\prime}$ than $g\left(C_{j}\right)$ whenever $i<j$. The standard word of $\sigma$, denoted by $W(\sigma)$, is $W\left(C_{1}\right) \cdots W\left(C_{c(\sigma)}\right)$.

Let $D$ be a braid diagram and $v$ be a vertex of $D$. Let us denote by $e_{1}, e_{2}$ the edges incident into $v$, and $e_{3}, e_{4}$ the edges incident from $v$ as described in Figure 5. We call left tangence at $v$ the ordered pair $\left(e_{1}, e_{3}\right)$ and right tangence at $v$ the ordered pair $\left(e_{2}, e_{4}\right)$. The pairs $\left(e_{1}, e_{4}\right)$ and $\left(e_{2}, e_{3}\right)$ will be called crossings at $v$. We call passage any ordered pair of edges that is either a tangence or a crossing at some vertex, or is of the form $(e, e)$ for some free loop $e$. 


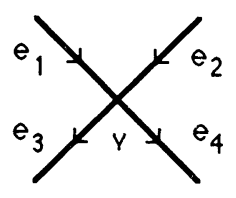

Figure 5

A circuit partition of $D$ is a permutation $\pi$ of its edge-set $E$ such that for every edge $e,(e, \pi(e))$ is a passage. The cycles of $\pi$ will be called circuits. A vertex $v$ is a tangence (respectively, crossing) point of $\pi$ if when $e$ is an edge incident into $v,(e, \pi(e))$ is a tangence (respectively, crossing) at $v$. Note that a circuit partition is determined uniquely by the corresponding status of the different vertices (tangence or crossing point). We shall denote by $t(\pi)$ the number of tangence points of $\pi$ and by $t^{\prime}(\pi)$ the number of such points $v$ with $s(v)=-1$.

The set of return edges of $D$, ordered by index, is an ordered transversal of every circuit partition. Thus, we may associate to every circuit partition $\pi$ its standard word with respect to this ordered transversal, which we denote by $W(\pi)$. Then for a vertex $v$, let $e$ be the leftmost letter of $W(\pi)$ that represents an edge incident into $v$. The first passage of $\pi$ at $v$ is $(e, \pi(e))$. The circuit partition $\pi$ of $D$ will be said to be admissible at the vertex $v$ if $s(v)=1$ and the first passage at $v$ is not a right tangence, or $s(v)=-1$ and the first passage at $v$ is not a left tangence. Finally, $\pi$ will be said to be admissible if it is admissible at every vertex of $D$. We shall denote by $A(D)$ the set of admissible circuit partitions of $D$. Then we may state our main result as follows.

Proposition 3. For any braid diagram on $n$ strings $D$,

$$
P(D, z, a)=a^{1-n-w(D)} \sum_{\pi \in A(D)}(-1)^{t^{\prime}(\pi)} z^{t(\pi)}\left(\left(a^{2}-1\right) z^{-1}\right)^{c(\pi)-1} .
$$

Before proving this result, we shall introduce a reformulation that will be easier to study.

3.2. Labellings, states, and transfer matrices. Let $D=(V, E, s)$ be a braid diagram on $n$ strings associated to the braid word on $n$ strings $m=m_{1} \cdots m_{k}$ as described in $\S 2.1$. Let $Q=\left\{p_{i, j} \mid i=0, \ldots, k ; j=1, \ldots, n\right\}$. For $i=$ $0, \ldots, k$ we denote by $Q_{i}$ the set $\left\{p_{i, j} \mid j=1, \ldots, n\right\}$ and we define the index of $p_{i, j}$ as equal to $j$.

We define a labelling of $D$ as a mapping $f$ from $Q$ to $\{1, \ldots, n\}$ that satisfies the following properties:

- $f\left(Q_{i}\right)=\{1, \ldots, n\}$ for $i=0, \ldots, k$.

- For $i=1, \ldots, k$, if $m_{i}$ equals $s_{r}$ or $s_{r}^{-1}, f\left(p_{i, j}\right)=f\left(p_{i-1, j}\right)$ for $j \in\{1, \ldots, n\}-\{r, r+1\}$. If $m_{i}=\varepsilon, f\left(p_{i, j}\right)=f\left(p_{i-1, j}\right)$ for $j=1, \ldots, n$. 
We shall denote by $L(D)$ the set of labellings of $D$.

To every circuit partition $\pi$ of $D$ we associate a labelling $f_{\pi}$ as follows. Assume that when we travel along the edge $e$ of $D$ from its initial end to its terminal end (or from an arbitrary point to the same point if $e$ is a free loop), we encounter successively the points $q_{1}, \ldots, q_{h}$ of $Q$ in this order. Then we denote by $q(e)$ the word $q_{1} \cdots q_{h}$ on the alphabet $Q$. Let us define the permutation $\pi^{\prime}$ of $Q$, which will be said associated to $\pi$, as follows. Each letter of $Q$ belongs to a unique word $q(e)$. Its image under $\pi^{\prime}$ is the next letter of $q(e)$ if it exists or the first letter of $q(\pi(e))$ otherwise. Clearly $c\left(\pi^{\prime}\right)=c(\pi)$. The set $Q_{0}$, ordered by index, is an ordered transversal of $\pi^{\prime}$. Let $W\left(\pi^{\prime}\right)$ be the standard word of $\pi^{\prime}$ with respect to this ordered transversal. This word has a unique factorization $Y_{1} \cdots Y_{n}$ such that, for $j=1, \ldots, n$, the first letter of $Y_{j}$ is in $Q_{0}$, the last letter of $Y_{j}$ is in $Q_{k}$, and the other letters of $Y_{j}$ are neither in $Q_{0}$ nor in $Q_{k}$. Consider the mapping from $Q$ to $\{1, \ldots, n\}$ that for $j=1, \ldots, n$ assigns to every element of $Y_{j}$ the value $j$. It is easy to check that this mapping is a labelling of $D$, which we denote by $f_{\pi}$.

Assume that $m_{i}$ equals $s_{r}$ or $s_{r}^{-1}$, and let $v_{i}$ be the vertex of $D$ corresponding to $m_{i}$. Clearly if $v_{i}$ is a tangence (respectively, crossing) point of $\pi,\left(f_{\pi}\left(p_{i, r}\right), f_{\pi}\left(p_{i, r+1}\right)\right)=\left(f_{\pi}\left(p_{i-1, r}\right), f_{\pi}\left(p_{i-1, r+1}\right)\right)$ (respectively, $\left(f_{\pi}\left(p_{i, r}\right)\right.$, $\left.\left.f_{\pi}\left(p_{i, r+1}\right)\right)=\left(f_{\pi}\left(p_{i-1, r+1}\right), f_{\pi}\left(p_{i-1, r}\right)\right)\right)$. It follows that the mapping $\pi \rightarrow f_{\pi}$ from the set of circuit partitions of $D$ to $L(D)$ is one-to-one. For a labelling $f$ let $\rho(f)$. be equal to zero if $f$ is not in the image of this mapping, and to $\left(\left(a^{2}-1\right) z^{-1}\right)^{c(\pi)-1}$ if $f=f_{\pi}$. We call $\rho(f)$ the return weight of $f$.

We now observe that if the first passage of $\pi$ at $v_{i}$ is $(e, \pi(e)), e$ contains the element of $\left\{p_{i-1, r}, p_{i-1, r+1}\right\}$ on which $f_{\pi}$ takes the smallest value. This leads us to define the interaction of the labelling $f$ with the vertex $v_{i}$, which we denote by $\left\langle v_{i} \mid f\right\rangle$, as follows. If $\left(f\left(p_{i, r}\right), f\left(p_{i, r+1}\right)\right)=\left(f\left(p_{i-1, r+1}\right), f\left(p_{i-1, r}\right)\right)$, then $\left\langle v_{i} \mid f\right\rangle=1$. If $\left(f\left(p_{i, r}\right), f\left(p_{i, r+1}\right)\right)=\left(f\left(p_{i-1, r}\right), f\left(p_{i-1, r+1}\right)\right)$, we distinguish two cases.

- If $s\left(v_{i}\right)=1,\left\langle v_{i} \mid f\right\rangle=z$ if $f\left(p_{i-1, r}\right)<f\left(p_{i-1, r+1}\right)$ and $\left\langle v_{i} \mid f\right\rangle=0$ if $f\left(p_{i-1, r}\right)>f\left(p_{i-1, r+1}\right)$.

- If $s\left(v_{i}\right)=-1,\left\langle v_{i} \mid f\right\rangle=0$ if $f\left(p_{i-1, r}\right)<f\left(p_{i-1, r+1}\right)$ and $\left\langle v_{i} \mid f\right\rangle=-z$ if $f\left(p_{i-1, r}\right)>f\left(p_{i-1, r+1}\right)$.

We write $\langle D \mid f\rangle=\prod_{i=1, \ldots, k}\left\langle v_{i} \mid f\right\rangle$ and we set this product as equal to 1 if $D$ has no vertices.

Now we may reformulate Proposition 3 as follows.

Proposition 4. For any braid diagram on $n$ strings $D$,

$$
P(D, z, a)=a^{1-n-w(D)} \sum_{f \in L(D)}\langle D \mid f\rangle \rho(f) .
$$

Proof of the equivalence with Proposition 3. The mapping $\pi \rightarrow f_{\pi}$ defines a bijection from $A(D)$ to the set of labellings $f$ with $\langle D \mid f\rangle \rho(f) \neq 0$. Moreover for every $\pi$ in $A(D),\left\langle D \mid f_{\pi}\right\rangle=(-1)^{t^{\prime}(\pi)} z^{t(\pi)}$ and $\rho\left(f_{\pi}\right)=\left(\left(a^{2}-1\right) z^{-1}\right)^{c(\pi)-1}$. 
We now reformulate Proposition 4 in matrix terms.

We first show that the return weight of a labelling $f$ is determined by the restrictions of $f$ to $Q_{0}$ and $Q_{k}$. The labelling $f$ being given, let $\pi^{\prime}$ be the permutation of $Q$ that for $i=0, \ldots, k-1$ associates to every element of $Q_{i}$ the unique element of $Q_{i+1}$ with the same $f$-value, and associates to every element of $Q_{k}$ the unique element of $Q_{0}$ with the same index. Clearly $\pi^{\prime}$ is associated to a unique circuit partition $\pi$ of $D$, which has the property that $\rho(f) \neq 0$ if and only if $f=f_{\pi}$. This will be the case if and only if $f$ and $f_{\pi}$ coincide on $Q_{0}$. Now let $\sigma$ be the permutation of $Q_{0}$ that for $j=1, \ldots, n$ associates to $p_{0, j}$ the unique point $p_{0, h}$ such that $f\left(p_{k, h}\right)=f\left(p_{0, j}\right)$. Note that $c(\sigma)=c\left(\pi^{\prime}\right)=c(\pi)$. The set $Q_{0}$ ordered by index is an ordered transversal of $\sigma$. We denote by $W(\sigma)$ the standard word of $\sigma$ with respect to this ordered transversal. Then it is easy to see that $W(\sigma)$ can be obtained from $W\left(\pi^{\prime}\right)=$ $Y_{1} \cdots Y_{n}$ by retaining only the first letter of each factor $Y_{j}$. Hence, denoting by $f(W(\sigma))$ the word obtained from $W(\sigma)$ by replacing each letter by its image under $f, \rho(f)=\left(\left(a^{2}-1\right) z^{-1}\right)^{c(\sigma)-1}$ if $f(W(\sigma))$ is equal to the word $1 \cdots n$, and $\rho(f)=0$ otherwise.

Let us call state of $D$ a sequence $g=\left(g_{i}, i=0, \ldots, k\right)$, where each $g_{i}$ is a permutation of $\{1, \ldots, n\}$, which satisfies the following properties for $i=1, \ldots, k$ :

- If $m_{i}=\varepsilon, g_{i}=g_{i-1}$.

- If $m_{i}$ equals $s_{r}$ or $s_{r}^{-1}, g_{i}=g_{i-1}$ or $g_{i}=g_{i-1} \circ \tau_{r}$, where $\tau_{r}$ is the transposition $(r, r+1)$.

We denote by $S(D)$ the set of states of $D$. For $i=0, \ldots, k$ let $\beta_{i}$ be the bijection from $\{1, \ldots, n\}$ to $Q_{i}=\left\{p_{i, j} \mid j=1, \ldots, n\right\}$ defined by $\beta_{i}(j)=p_{i, j}(j=1, \ldots, n)$. Let $f$ be a labelling of $D$. The sequence $g=\left(g_{i}, i=0, \ldots, k\right)$, where $g_{i}=f \circ \beta_{i}$, is easily seen to be a state that we denote by $\gamma(f)$. Clearly the mapping $\gamma$ is a bijection from $L(D)$ to $S(D)$. Let $g$ be a state of $D$. We have seen that $\rho\left(\gamma^{-1}(g)\right)$ is determined by the restrictions of $\gamma^{-1}(g)$ to $Q_{0}$ and $Q_{k}$. Hence, it is determined by $g_{0}$ and $g_{k}$ in a similar fashion. More precisely, we introduce a return weight matrix $R_{n}$ (also denoted by $R$ if the context is clear) whose rows and columns are indexed by the group $S_{n}$ of permutations of $\{1, \ldots, n\}$. Let $\lambda, \mu$ be two permutations of $\{1, \ldots, n\}$. Consider the permutation $\nu=\lambda^{-1} \circ \mu$. Let $W(\nu)$ be the standard word of $\nu$ with respect to its ordered transversal $\{1, \ldots, n\}$ (with its natural order). We set $R(\lambda, \mu)=\left(\left(a^{2}-1\right) z^{-1}\right)^{c(\nu)-1}$ if $W(\nu)$ is equal to the word $\mu^{-1}(1) \cdots \mu^{-1}(n)$, and $R(\lambda, \mu)=0$ otherwise. Then it is easy to check that for every state $g=\left(g_{i}, i=0, \ldots, k\right), \rho\left(\gamma^{-1}(g)\right)=R\left(g_{k}, g_{0}\right)$.

Now for $r=1, \ldots, n-1$ we introduce transfer matrices $T_{n, r}^{+}$and $T_{n, r}^{-}$(or, more briefly, $T_{r}^{+}$and $T_{r}^{-}$if this notation creates no ambiguity) whose rows and columns are indexed by $S_{n}$. For $\lambda$ and $\mu$ in $S_{n}$, the corresponding matrix elements $T_{r}^{+}(\lambda, \mu)$ and $T_{r}^{-}(\lambda, \mu)$ are defined as follows. If $\mu=\lambda \circ \tau_{r}$, then 
$T_{r}^{+}(\lambda, \mu)=T_{r}^{-}(\lambda, \mu)=1$. If $\mu=\lambda$ and $\lambda(r)<\lambda(r+1)$ then $T_{r}^{+}(\lambda, \mu)=z$ and $T_{r}^{-}(\lambda, \mu)=0$. If $\mu=\lambda$ and $\lambda(r)>\lambda(r+1)$, then $T_{r}^{+}(\lambda, \mu)=0$ and $T_{r}^{-}(\lambda, \mu)=-z$. In all other cases, $T_{r}^{+}(\lambda, \mu)=T_{r}^{-}(\lambda, \mu)=0$.

Let now $f$ be a labelling of $D$ and let $\gamma(f)=g=\left(g_{i}, i=0, \ldots, k\right)$ be the corresponding state. It is easy to check that if $m_{i}$ equals $s_{r}$ (respectively, $s_{r}^{-1}$ ), denoting by $v_{i}$ the vertex of $D$ corresponding to $m_{i}$, the interaction $\left\langle v_{i} \mid f\right\rangle$ is equal to $T_{r}^{+}\left(g_{i-1}, g_{i}\right)$ (respectively, $\left.T_{r}^{-}\left(g_{i-1}, g_{i}\right)\right)$. Let $I$ be the identity matrix with rows and columns indexed by $S_{n}$. For all $i=1, \ldots, k$, let $M_{i}$ be the matrix $I$ if $m_{i}=\varepsilon, T_{r}^{+}$if $m_{i}=s_{r}, T_{r}^{-}$if $m_{i}=s_{r}^{-1}$. Then if $g=\left(g_{i}, i=0, \ldots, k\right)$ is a state, $\prod_{i=1, \ldots, k} M_{i}\left(g_{i-1}, g_{i}\right)=\left\langle D \mid \gamma^{-1}(g)\right\rangle$. Moreover if the sequence $g=\left(g_{i}, i=0, \ldots, k\right)$, where the $g_{i}$ belong to $S_{n}$, is not a state, $\prod_{i=1, \ldots, k} M_{i}\left(g_{i-1}, g_{i}\right)$ equals 0 . We now obtain the following matrix form of Proposition 4.

Proposition 5. For any braid diagram on $n$ strings $D$ associated to the braid word on $n$ strings $m=m_{1} \cdots m_{k}$,

$$
P(D, z, a)=a^{1-n-w(D)} \operatorname{Trace}\left(\left(\prod_{i=1, \ldots, k} M_{i}\right) R\right) .
$$

Proof of the equivalence with Proposition 4. Since $\gamma$ is a bijection from $L(D)$ to $S(D)$, writing $g=\left(g_{i}, i=0, \ldots, k\right)$ for every state $g$, we have

$$
\begin{aligned}
\sum_{f \in L(D)}\langle D \mid f\rangle \rho(f) & =\sum_{g \in S(D)}\left\langle D \mid \gamma^{-1}(g)\right\rangle \rho\left(\gamma^{-1}(g)\right) \\
& =\sum_{g \in S(D)}\left(\prod_{i=1, \ldots, k} M_{i}\left(g_{i-1}, g_{i}\right)\right) R\left(g_{k}, g_{0}\right) .
\end{aligned}
$$

The similar summation extended to all sequences $g=\left(g_{i}, i=0, \ldots, k\right)$, where the $g_{i}$ belong to $S_{n}$, has the same value, since $\prod_{i=1, \ldots, k} M_{i}\left(g_{i-1}, g_{i}\right)$ is zero if $g$ is not a state. This last expression is the trace of the matrix $\left(\left(\prod_{i=1, \ldots, k} M_{i}\right) R\right)$.

3.3. Proof of Propositions 3, 4, and 5. Let $D$ be a braid diagram on $n$ strings associated to the braid word on $n$ strings $m=m_{1} \cdots m_{k}$. We want to show that

$$
P(D, z, a)=a^{1-n-w^{\prime}(D)} \operatorname{Trace}\left(\left(\prod_{i=1, \ldots, k} M_{i}\right) R\right) .
$$

Let $G L\left(S_{n}, K\right)$ be the multiplicative group of invertible square matrices with coefficients in $K$ whose rows and columns are indexed by $S_{n}$. It is easy to check that for $r=1, \ldots, n-1, T_{r}^{+} T_{r}^{-}=I$ (it is enough to do this for $n=2)$. Similarly one can easily check that for $r=1, \ldots, n-2, T_{r}^{+} T_{r+1}^{+} T_{r}^{+}=$ $T_{r+1}^{+} T_{r}^{+} T_{r+1}^{+}$(clearly we can restrict our attention to the case $n=3$ ). The 
commutation relations $T_{r}^{+} T_{r^{\prime}}^{+}=T_{r^{\prime}}^{+} T_{r}^{+}$for $\left|r^{\prime}-r\right| \geq 2$ are immediate. It follows that for every integer $n \geq 1$, there exists a representation $\varphi_{n}: B_{n} \rightarrow$ $G L\left(S_{n}, K\right)$ such that $\varphi_{n}\left(s_{r}\right)=T_{n, r}^{+}$and $\varphi_{n}\left(s_{r}^{-1}\right)=T_{n, r}^{-}$(these representations will easily be seen to be essentially identical to the classical representations of Hecke algebras described, for instance, in [8].) For every positive integer $n$, let us define a mapping $F_{n}$ from $B_{n}$ to $K$ as follows: for every $b$ in $B_{n}, F_{n}(b)=$ $a^{1-n-w(b)} \operatorname{Trace}\left(\varphi_{n}(b) R_{n}\right)$. It remains to show that for every braid diagram on $n$ strings $D$ that is a closure of the braid $(b, n), F_{n}(b)=P(D, z, a)$. This will be done by proving that properties (i), (ii), and (iii) of Proposition 2 hold for $F_{n}$. Property (iii) reduces to $\operatorname{Trace}\left(R_{1}\right)=1$, which is immediate. One easily checks that $T^{+}-T^{-}=z I$. Hence for $i=1, \ldots, n-1, \varphi_{n}\left(b s_{i} b^{\prime}\right)-\varphi_{n}\left(b s_{i}^{-1} b^{\prime}\right)=$ $z \varphi_{n}\left(b b^{\prime}\right)$. Since $w\left(b b^{\prime}\right)=w\left(b s_{i} b^{\prime}\right)-1=w\left(b s_{i}^{-1} b^{\prime}\right)+1$, property (ii) follows.

Now for the study of property (i) it is convenient to come back to the formulation in terms of circuit partitions. Clearly property (i) is equivalent to the following:

(i') Let $m$ and $m^{\prime}$ be two braid words on $n$ strings and let $D$ be the braid diagram on $n$ strings associated to $\mathrm{mm}^{\prime}$. Let $D^{1}$ (respectively, $D^{2}$ ) be the braid diagram on $n+1$ strings associated with $m s_{n} m^{\prime}$ (respectively, $m s_{n}^{-1} m^{\prime}$ ). Then

$$
\sum_{\pi \in A(D)} C(\pi)=a^{-2} \sum_{\pi \in A\left(D^{1}\right)} C(\pi)=\sum_{\pi \in A\left(D^{2}\right)} C(\pi),
$$

where $C(\pi)=(-1)^{t^{\prime}(\pi)} z^{t(\pi)}\left(\left(a^{2}-1\right) z^{-1}\right)^{c(\pi)-1}$.

We denote by $v^{1}$ (respectively, $v^{2}$ ) the vertex of $D^{1}$ (respectively, $D^{2}$ ) corresponding to $s_{n}$ (respectively, $\left.s_{n}^{-1}\right)$. Let $V^{i}$ be the vertex-set of $D^{i} \quad(i=$ $1,2)$. We identify $V^{i}-\left\{v^{i}\right\}$ with the vertex-set $V$ of $D$. Let $\pi$ be a circuit partition of $D$. A circuit partition of $D^{i}$ will be said to be compatible with $\pi$ if every vertex of $V$ that is a tangence (respectively, crossing) point of this partition is also a tangence (respectively, crossing) point of $\pi$. We denote by $\tau_{i}(\pi)$ (respectively, $\kappa_{i}(\pi)$ ) the unique circuit partition of $D^{i}$ compatible with $\pi$ that has $v^{i}$ as a tangence (respectively, crossing) point. Every circuit partition of $D^{i}$ is equal either to $\tau_{i}(\pi)$ or to $\kappa_{i}(\pi)$ for a unique circuit partition $\pi$ of $D$. Moreover, it is easy to see that $\tau_{1}(\pi), \kappa_{1}(\pi), \kappa_{2}(\pi)$ are admissible if and only if $\pi$ is admissible, and that $\tau_{2}(\pi)$ is never admissible. Hence

$$
\sum_{\pi \in A\left(D^{\prime}\right)} C(\pi)=\sum_{\pi \in A(D)}\left(C\left(\tau_{1}(\pi)\right)+C\left(\kappa_{1}(\pi)\right)\right)
$$

and

$$
\sum_{\pi \in A\left(D^{2}\right)} C(\pi)=\sum_{\pi \in A(D)} C\left(\kappa_{2}(\pi)\right) .
$$

Thus, to prove $\left(\mathrm{i}^{\prime}\right)$ it is enough to prove that for every circuit partition $\pi$ of $D$, $C(\pi)=C\left(\kappa_{2}(\pi)\right)$ and $C(\pi)=a^{-2}\left(C\left(\tau_{1}(\pi)\right)+C\left(\kappa_{1}(\pi)\right)\right)$. The first equality is 
immediate since the parameters $t, t^{\prime}$, and $c$ take the same values for $\pi$ and $\kappa_{2}(\pi)$. For the second equality, we note that $t\left(\tau_{1}(\pi)\right)=t(\pi)+1, t^{\prime}\left(\tau_{1}(\pi)\right)=$ $t^{\prime}(\pi), c\left(\tau_{1}(\pi)\right)=c(\pi)+1, t\left(\kappa_{1}(\pi)\right)=t(\pi), t^{\prime}\left(\kappa_{1}(\pi)\right)=t^{\prime}(\pi)$, and $c\left(\kappa_{1}(\pi)\right)=$ $c(\pi)$. Hence, $C\left(\tau_{1}(\pi)\right)=z\left(\left(a^{2}-1\right) z^{-1}\right) C(\pi)$ and $C\left(\kappa_{1}(\pi)\right)=C(\pi)$. The result follows immediately.

\section{SOME CONSEQUENCES}

4.1. Degrees in the variable $a$. It follows from Proposition 3 that $P(D, z, a)$ is a polynomial (with no negative exponents) in the variables $z$ and $\left(a^{2}-1\right) z^{-1}$, multiplied by a factor $a^{1-n-w(D)}$. A similar result (Theorem 1.8 ) is proved by inductive methods in [6]. See also [19] for a related work on arbitrary diagrams. An important consequence is that if we denote by $e_{\min }(D)$ (respectively, $\left.e_{\max }(D)\right)$ the minimum (respectively, maximum) degree of $P(D, z, a)$ in the variable $a$, the following "Morton-Franks-Williams inequalities" hold:

(i) $1-n-w(D) \leq e_{\min }(D) \leq e_{\max }(D) \leq n-1-w(D)$,

(ii) $n \geq 1+\left(\left(e_{\max }(D)-e_{\min }(D)\right) / 2\right)$.

The inequalities (i) follow from Proposition 3, since $1 \leq c(\pi) \leq n$ for every circuit partition $\pi$. The inequality (ii) is an immediate consequence of (i) and has proved to be useful in the study of the braid index. It has been observed (see, for instance, [12]) that equality occurs frequently in (ii). This is the case if and only if both inequalities in (i) hold with equality. The following easy consequence of Proposition 3 characterizes this situation in terms of circuit partitions.

Proposition 6. For any braid diagram on $n$ strings $D:(1) 1-n-w(D) \leq$ $e_{\text {min }}(D)$ with equality iff $\sum_{\pi \in A(D)}(-1)^{t^{\prime}(\pi)-c(\pi)+1} z^{t(\pi)-c(\pi)+1} \neq 0$. (2) $e_{\max }(D) \leq$ $n-1-w(D)$ with equality iff $\sum_{\pi \in A(D), c(\pi)=n}(-1)^{t^{\prime}(\pi)} z^{t(\pi)} \neq 0$.

A braid diagram is said to be positive (respectively, negative) if every vertex has positive (respectively, negative) sign. In answer to a question of Franks and Williams, Morton and Short exhibit in [20] a positive braid for which the inequality (ii) is strict. However, we have the following result.

Proposition 7. (1) For any negative braid diagram on $n$ strings $D, e_{\min }(D)=$ $1-n-w(D)$. (2) For any positive braid diagram on $n$ strings $D, e_{\max }(D)=$ $n-1-w(D)$.

Proof. (1) If $D$ is negative, for every circuit partition $\pi$ of $D, t^{\prime}(\pi)=t(\pi)$. Since the circuit partition with no tangence points is admissible, the result follows from Proposition 6, part (1).

(2) If $D$ is positive, for every circuit partition $\pi$ of $D, t^{\prime}(\pi)=0$. Moreover, the circuit partition for which all vertices are tangence points is admissible and has $n$ circuits. The result now follows from Proposition 6, part (2).

Remark. Let $D^{\sim}$ denote the mirror image of $D$, that is, the braid diagram obtained from $D$ by changing the signs of all vertices. It follows immediately 
from Proposition 1 that $P\left(D^{\sim}, z, a\right)=P\left(D,-z, a^{-1}\right)$. Hence, $e_{\min }\left(D^{\sim}\right)=$ $-e_{\max }(D)$ and we obtain directly the equivalence of parts (1) and (2) of Proposition 7 .

4.2. Degrees in the variable $z$. Let $D$ be a braid diagram on $n$ strings with vertex-set $V$. By Proposition 3,

$$
P(D, z, a)=a^{1-n-w(D)} \sum_{\pi \in A(D)}(-1)^{t^{\prime}(\pi)}\left(a^{2}-1\right)^{c(\pi)-1} z^{t(\pi)-c(\pi)+1} .
$$

Let $\pi_{0}$ (respectively, $\pi_{1}$ ) be the circuit partition of $D$ for which all vertices are crossing (respectively, tangence) points. Then $t\left(\pi_{0}\right)=0, t\left(\pi_{1}\right)=|V|$, $c\left(\pi_{1}\right)=n$ and $c\left(\pi_{0}\right)$ is the number of components of $D$, which we denote by $k(D)$. If the circuit partition $\pi^{\prime}$ is obtained from the circuit partition $\pi$ by changing exactly one crossing point into a tangence point, $t\left(\pi^{\prime}\right)=t(\pi)+1$ and $c\left(\pi^{\prime}\right) \leq c(\pi)+1$, so that $t\left(\pi^{\prime}\right)-c\left(\pi^{\prime}\right) \geq t(\pi)-c(\pi)$. It follows that for every circuit partition $\pi$ of $D, 1-k(D) \leq t(\pi)-c(\pi)+1 \leq|V|-n+1$. Moreover, the coefficient of $z^{1-k(D)}$ in $P(D, z, a)$ is, up to the multiplicative factor $a^{1-n-w(D)}\left(a^{2}-1\right)^{k(D)-1}$, equal to

$$
\sum_{\pi \in A(D), c(\pi)-t(\pi)=k(D)}(-1)^{t^{\prime}(\pi)}\left(a^{2}-1\right)^{t(\pi)} .
$$

The constant term of this polynomial in the variable $a^{2}-1$ is nonzero, since $\pi_{0}$ is admissible. Hence, we obtain the following result.

Proposition 8. Let $D$ be a diagram.

(1) The minimum degree of $P(D, z, a)$ in the variable $z$ equals $1-k(D)$.

(2) If $D$ is a braid diagram on $n$ strings with vertex-set $V$, the maximum degree of $P(D, z, a)$ in the variable $z$ is not greater than $|V|-n+1$.

Part (1) of Proposition 8 appears in [18], and a generalization of part (2) to all diagrams is given in [19]. In both cases the method of proof is by induction using the definition of Proposition 1.

4.3. A simple model for the Alexander-Conway poiynomial. The AlexanderConway polynomial of $D[1,5]$, which we denote by $\nabla(D, z)$, is equal to $P(D, z, 1)$. Thus, the following result is a special case of Proposition 3 .

Proposition 9. For any braid diagram $D$,

$$
\nabla(D, z)=\sum_{\pi \in A(D), c(\pi)=1}(-1)^{t^{\prime}(\pi)} z^{t(\pi)} .
$$

As a consequence, if $D$ is a positive braid diagram, $\nabla(D, z)$ has positive integer coefficients. More precisely, the coefficient of $z^{k}$ in $\nabla(D, z)$ is the number of admissible circuit partitions of $D$ with exactly one circuit and exactly $k$ tangent points. 
4.4. On the number of admissible circuit partitions. The author is grateful to the referee for proposing the study of the number of admissible circuit partitions of a braid diagram as a function of its number of vertices. This suggestion is motivated by the observation that for the Jones polynomial, Kauffman's state model [15] associates to a diagram with $p$ vertices an expansion involving $2^{p}$ terms, whereas the circuit partition expansion would involve fewer terms in general.

A first remark is that by Proposition 3, for any positive braid diagram $D$ on $n$ strings with $p$ vertices, $|A(D)|=(\sqrt{ } 2)^{n+p-1} P(D, 1, \sqrt{ } 2)$. This equality was used in some of the computations reported here.

It is not difficult to see that the average number of admissible circuit partitions of a braid diagram with $p$ vertices is $(3 / 2)^{p}$. More precisely, let $D=(V, E, s)$ be a braid diagram with $|V|=p$, and for $S \subseteq V$ let $D_{S}$ be the diagram obtained from $D$ by changing the signs of the vertices of $S$. Since a circuit partition of the graph $(V, E)$ with exactly $x$ crossing points is admissible for exactly $2^{x}$ diagrams of the form $D_{S}$, it is clear that $\sum_{S \subseteq V}\left|A\left(D_{S}\right)\right|=\sum_{X \subseteq V} 2^{|X|}=3^{p}$, and hence the average number of admissible circuit partitions of a diagram $D_{S}$ is $(3 / 2)^{p}$. We may interpret this number as a theoretical measure of the average time complexity of the computation of the homfly polynomial of a closed braid with $p$ vertices using the circuit partition model.

A similar measure of the worst case time complexity of this computation would be given by the maximum possible number $A(n, p)$ of admissible circuit partitions of a braid diagram on $n$ strings with $p$ vertices. Let $f_{p}$ be the $p$ th Fibonacci number defined by the recurrence relation $f_{p}=f_{p-1}+f_{p-2}$ with the initial conditions $f_{0}=f_{1}=1$, or equivalently by the formula

$$
f_{p}=(1 / \sqrt{ } 5)((1+\sqrt{ } 5) / 2)^{p+1}-(1 / \sqrt{ } 5)((1-\sqrt{ } 5) / 2)^{p+1} \text {. }
$$

It is not difficult to show that $A(2, p)=f_{p+1}$ (this maximum being given by the positive braid diagram corresponding to the braid word $s_{1}^{p}$ ). In general, for $p \leq n-1, A(n, p)=2^{p}$ (this is given by the braid words $\left.s_{1} \cdots s_{p}\right)$. For $p \geq n$, the braid word $s_{1}^{p-n+2} s_{2} \cdots s_{n-1}$ gives $A(n, p) \geq 2^{n-2} f_{p-n+3}$, and there is experimental evidence that equality might hold. Thus it seems reasonable to conjecture that for every fixed $n, A(n, p)$ is equivalent when $p \rightarrow \infty$ to $c_{n}((1+\sqrt{ } 5) / 2)^{p}$ for some constant $c_{n}$.

The author would like to thank Michel Mollard for helpful discussions.

\section{REFERENCES}

1. J. W. Alexander, Topological invariants of knots and links, Trans. Amer. Math. Soc. 30 (1928), 275-306.

2. C. Berge, Graphes et hypergraphes, Dunod, Paris, 1974.

3. J. A. Bondy and U. S. R. Murty, Graph theory with applications, Macmillan, London, 1976.

4. G. Burde and H. Zieschang, Knots, De Gruyter, Berlin and New York, 1985.

5. J. H. Conway, An enumeration of knots and links and some of their algebraic properties, Computational Problems in Abstract Algebra, Pergamon, New York, 1970, pp. 329-358. 
6. J. Franks and R. F. Williams, Braids and the Jones polynomial, Trans. Amer. Math. Soc. 303 (1987), 97-108.

7. P. Freyd, D. Yetter, J. Hoste, W. B. R. Lickorish, K. Millett, and A. Ocneanu, A new polynomial invariant of knots and links, Bull. Amer. Math. Soc. 12 (1985), 239-246.

8. P. de la Harpe, M. Kervaire, and C. Weber, On the Jones polynomial, Enseign. Math. 32 (2) (1986), 271-235.

9. J. Hoste, A polynomial invariant of knots and links, Pacific J. Math. 124 (1986), 295-320.

10. F. Jaeger, Composition products and models for the homfly polynomial, Enseign. Math. 32 (1989), 323-361.

11. ___ A combinatorial model for the homfly polynomial, Europ. J. Combinatorics (to appear).

12. V. F. R. Jones, Hecke algebra representations of braid groups and link polynomials, Ann. of Math. 126 (1987), 335-388.

13. __ Notes on a talk in Atiyah's seminar, November 1986.

14. L. H. Kauffman, Formal knot theory, Math. Notes, no. 30, Princeton Univ. Press, 1983.

15. __ State models and the Jones polynomial, Topology 26 (1987), 395-407.

16. State models for knot polynomials-An introduction, Proc. 1987 Winter Meeting of the Brasilian Mathematical Society.

17. _ Knots, abstract tensors and the Yang-Baxter equation, preprint.

18. W. B. R. Lickorish and K. Millett, A polynomial invariant of oriented links, Topology 26 (1987), 107-141.

19. H. R. Morton, Seifert circles and knot polynomials, Math. Proc. Cambridge Philos. Soc. 99 (1986), 107-109.

20. H. R. Morton and H. B. Short, The 2-variable polynomial of cable knots, Math. Proc. Cambridge Philos. Soc. 101 (1987), 267-278.

21. K. Murasugi, Jones polynomials and classical conjectures in knot theory, Topology 26 (1987), 187-194.

22. J. H. Przytycki and P. Traczyk, Invariants of links of Conway type, Kobe J. Math. 4 (1987), 115-139.

23. M. Thistlethwaite, A spanning tree expansion of the Jones polynomial, Topology 26 (1987), 297-309.

24. V. G. Turaev, The Yang-Baxter equation and invariants of links, Invent. Math. 92 (1988), 527-553.

25. P. Vogel, Représentations et traces des algèbres de Hecke, polynôme de Jones-Conway, Enseign. Math. 34 (1988), 333-356.

Laboratoire de Structures Discrètes et de Didactique, IMAG, BP 53X, 38041 Grenoble Cédex, France 